\title{
Systemic lupus erythematosus with subacute cutaneous manifestation - acute on chronic.
}

Systemic Lupus Erythematosus (SLE) presents as a continuum, with some patients demonstrating only cutaneous manifestations, such as Chronic Cutaneous Lupus Erythematosus (CCLE) or other more severe organ manifestations as in SLE. ${ }^{1}$ Between the two extremes of the spectrum can occur Subacute Cutaneous Lupus Erythematosus (SCLE). Although usually benign, $1 \%$ to $5 \%$ of patients with CCLE may develop SLE, and $25 \%$ of SLE patients may develop typical chronic discoid lesions at any time during the course of the disease. ${ }^{2,3,4,}$ Subacute Cutaneous Lupus Erythematosus presents with distinct clinical and serological characteristics. It occurs over areas of sun exposure and healing without scars and presents as intermittent recurrence with long periods of time without activity and without significant progression for systemic involvement. ${ }^{1}$ The classic presentation is annular or psoriasiform skin lesions located above the waist (chest, face, and back of the hands); ${ }^{5}$ The authors present a 48-year-old woman with Discoid Cutaneous Lupus (DCL) at 26 years of age characterized by erythematous plaques located in the nasal, malar and chest region, also diagnosed with Systemic Lupus Erythematosus 15 years (with arthralgia, lymphopenia and ANA (1/640), Anti SS-A (26 U/mL) and Anti SS-B (178 U/ $\mathrm{mL}$ ) and maintained hydroxychloroquine therapy for several years without disease activity. After years without manifestations, presented with erythematous plaques on the nose, cheeks, dorsal aspect of all fingers and toes with scaling, atrophy and scarring and confluent erythematous papules and plaques are visible in the chest area, those without significant scaling or atrophy. (Figure 1 and 2). Given the characteristics of the lesions and serious constitutional symptoms, it was assumed Subacute Cutaneous Lupus. For the refractoriness of the lesions after systemic corticoid therapy, it was performed intravenous immunoglobulins followed with rituximab cycle with a total resolution of the cutaneous manifestations without scarring sequelae and resolution of the inflammatory syndrome was resolved. The distinction between CCLE and SLE con be difficult. This case highlights this difficulty when patients presents with manifestations both from chronic and subacute forms. The correct distinction allows the institution of appropriate therapy, and so it is important to acknowledge each kind of manifestation in order to define the adequate diagnosis. In the present case, it evolved refractoriness to classic immunosuppressive therapy requiring the institution of other therapeutic alternatives.
Fig. 1. Erythematous plaques on the nose, left cheek and superior cutaneous lip, with areas of scaling, atrophy and scarring, particularly in the nasal tip. Confluent erythematous papules and plaques are visible in the chest area, without significant scaling or atrophy.

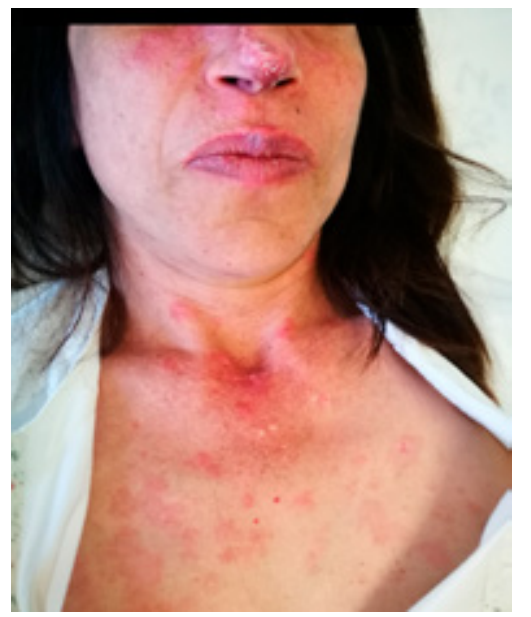

Fig. 2. Involvement of the dorsal aspect of all fingers and toes, with erythema, scaling and some crusts on the haluxes. Some atrophic erythematous and depigmented tiny macules are visible on the dorsal aspect of the hands and feet.

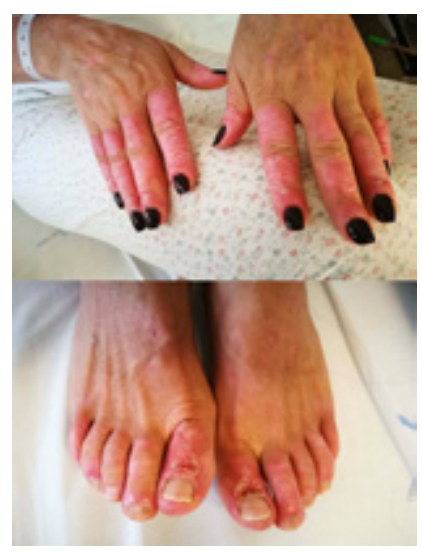

\section{References}

1. Costner IM, Sontheimer RD. Lupus erythematous. In: Klauswolff, Goldsmith LA, Katz SI, Gilchrist BA, Paller AS, Leffell DJ, editors. Fitz Patrick Dermatology in General Medicine. 7th ed. New York: McGraw Hill; 2008. pp. 1522-9.

2. Fitzpatrick TB, Johnson RA, Klaus W, Suurmond D. In colour atlas and synopsis of clinical dermatology, 4th ed. New York (NY): McGraw-Hill Companies;2001: 368-9.

3. Lahita RG. In systemic lupus erythematosus, 2nd ed. New York (NY): Churchill Livingstone; 1987: 620.

4. Grönhagen CM, Fored CM, Granath F, Nyberg F. Cutaneous lupus erythematosus and the association with systemic lupus erythematosus: a population-based cohort of 1088 patients in Sweden. Br J Dermatol. 2011;164(6):1335-1341.

5. Goodfield MJ, Jones SK, Vealae DJ. The connective tissue diseases. In: Burns T, Breathnach S, Cox N, Griffiths C, editors. Rooks Textbook of Dermatology. 8th ed. UK: Wiley Blackwell; 2010. pp. 51.21-3.

Diagnosis: Subacute Cutaneous Lupus

Juliana Lopes ${ }^{1}$, Isabel Almeida ${ }^{2,3}$

Internal Medicine Department - Centro Hospitalar do Porto

Emergency Department - Centro Hospitalar do Porto

Clinic Immunology Unit - Centro Hospitalar do Porto 